\title{
EGYPTE GRECO ROMAINE
}

\section{Nouveaux ostraca grees du Mons Claudianus}

$\mathrm{L}$

'ABSENCE de témoignages écrits postérieurs à la seconde moitié du ${ }^{\mathrm{II}^{\mathrm{e}}}$ siècle de notre ère - ils existent en revanche pour le Mons Porphyrites - conduisait Fitzler à penser que l'extraction du granit au Mons Claudianus s'était arrêtée vers la fin du règne d'Hadrien $\left({ }^{(}\right)$. Cette opinion, qui a longtemps fait autorité et que Guy Wagner et moi-même reprenions récemment $\left({ }^{2}\right)$, est néanmoins contredite par les résultats de l'enquête menée par Th. Kraus, J. Röder et W. Müller-Wiener sur les techniques d'extraction et sur l'architecture du camp principal $\left({ }^{3}\right)$. Sans doute l'équipe allemande a-t-elle cru détecter une phase d'abandon du site qui, d'après les critères considérés, serait intervenue entre le milieu du $\mathrm{II}^{\mathrm{e}}$ siècle et la fin $\mathrm{du} \mathrm{III}^{\mathrm{e}}\left(^{4}\right)$, mais le P. Oxy. 3243 (214/5), qui mentionne l'envoi de céréales depuis le Fayoum «à ceux qui sont en service dans les carrières du Porphyrites $t \mathrm{t}$ du Claudianus ", fournit un terminus a quo qui repousse l'arrêt provisoire de l'extraction bien après la fin du règne d'Hadrien. Il semble acquis en tout cas que le granit du Mons Claudianus était encore exploité au rv ${ }^{\mathrm{e}}$ siècle $\left(^{5}\right)$.

Aucun des ostraca étudiés ici n'est daté $\left({ }^{6}\right)$, mais la paléographie permet de les assigner aux deux premiers siècles de notre ère : comme

(1) K. FitzLER, Steinbrüche und Bergwerke im ptolemäischen und römischen Aegypten (1910), pp. 98-99.

(2) H. CuvignY, G. WAGNER, Ostraca grecs du Mons Claudianus. ZPE 62 (1986), pp. 63-73.

(3) Th. Kraus, J. RöDer, Mons Claudianus. Bericht über eine erste Erkundungsfahrt im März 1961. MDAIK 18 (1962), pp. 80-120. Th. Kraus, J. Röder, W. MüLden-WIENer, Mons Claudianus-Mons Porphyrites. Bericht über die zweite Forschungsreise 1964. MDAIK 22 (1967), pp. 108-205.

(4) $M D A I K 22$, pp. 118 et 145.

(5) $M D A I K 22$, p. 119 et n. 1 ; p. 145 . L'élargissement du camp, datable de la fin du $\mathrm{Im}^{\mathrm{e}}$ siècle ou du $\mathrm{Iv}^{\mathrm{e}}$, reflète des intentions ambitieuses, mais la carte des lits de carriers qui remontent à cette époque (l.c. 144), comparée avec celle du II/III ${ }^{\mathrm{e}}$ s., trahit une reprise de l'activité non tant modeste peut-être que de courte durée.

(6) Nous avions cru lire une date dans la lettre $Z P E 62$, p. $68, n^{\circ} 3$. Mais cette date était surprenante à plus d'un titre, puisqu'elle se trouvait en tête du texte et non à la fin, comme c'est l'usage dans les lettres, que le jour du mois était omis, et surtout qu'il s'agissait d'un mois macédonien, ce qui est exceptionnel dans les papyrus 
tous les textes (ostraca et inscriptions) trouvés sur le site et publiés à ce jour, ils sont donc contemporains de la première période d'occupation. Ils constituent un ensemble cohérent où les questions d'approvisionnement sont au premier plan (distributions massives de pain, octroi de vin à titre de pourboire, acheminement de l'eau). Peu sensible dans la précédente série d'ostraca, la présence de l'armée romaine que manifestent les inscriptions et l'implantation des édifices ${ }^{1}$ ) est ici complètement occultée : mis à part quelques noms latins sur lesquels on ne peut guère se fonder, nos documents ne parlent que de la population artisanale gréco-égyptienne qui devait être majoritaire $\left(^{2}\right)$.

1. LetTre. $10 \times 10 \mathrm{~cm}$. (fig. 1).

Ce texte s'inscrit négativement dans la catégorie banale des ordres de livraison ou de paiement, puisqu'il s'agit d'un contrordre. $2^{\mathrm{e}}$ moitié $\mathrm{du}$ $1^{\mathrm{er}}$ ou début du II $^{\mathrm{e}} \mathrm{s}$. d'après l'écriture : cf. $P$. Warr. 8 (pl. II, 86 p. C.) et P. Mil. Vogl. 264 (pl. II, 127 p.C.).

$\Pi \alpha_{\chi \nu o \tilde{v} \mu \iota \varsigma} \Pi \varepsilon \tau \varepsilon \chi \tilde{\omega} \nu \tau \iota$

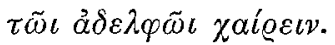

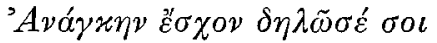

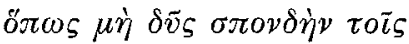

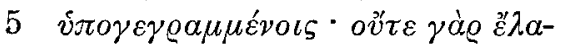

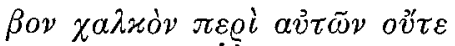

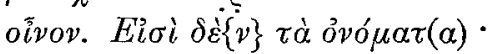

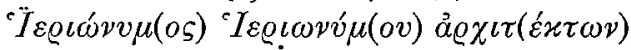

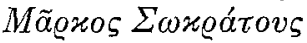

10 'Ioídwoos 'Q @ílwos.
$3 l . \delta \eta \lambda \tilde{\omega} \sigma \alpha \iota$
$4 \delta \tilde{v} \varsigma$ pour $\delta o \tilde{\iota}, l . \delta \tilde{\omega} \varsigma$

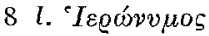
$\ddot{I} \varepsilon \varrho \iota \omega \nu v^{\mu}$

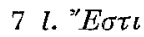
ovo $\mu \alpha^{\tau}$

"Pachnoumis à son frère Petechôn, salut. Je dois t'avertir de ne pas donner de prime aux personnes mentionnées ci-dessous ; je n'ai en effet

d'époque romaine. J'adhère entièrement à la suggestion de P. Sijpesteijn, pour qui il faut simplement lire $\Delta \circ v \pi \tilde{\alpha}$ ' $x \alpha i{ }^{\prime} \Delta \tilde{\eta} \circ \varsigma^{\prime}$ ( $l$. $\Delta \varepsilon \tilde{i} \sigma \varsigma$ ) (lettre à G. Wagner du 12 mars $86)$.

(1) MDAIK 22, p. 115.

(2) Comme ceux que j'ai publiés précédemment avec G. Wagner, ils appartiennent à une collection privée. 

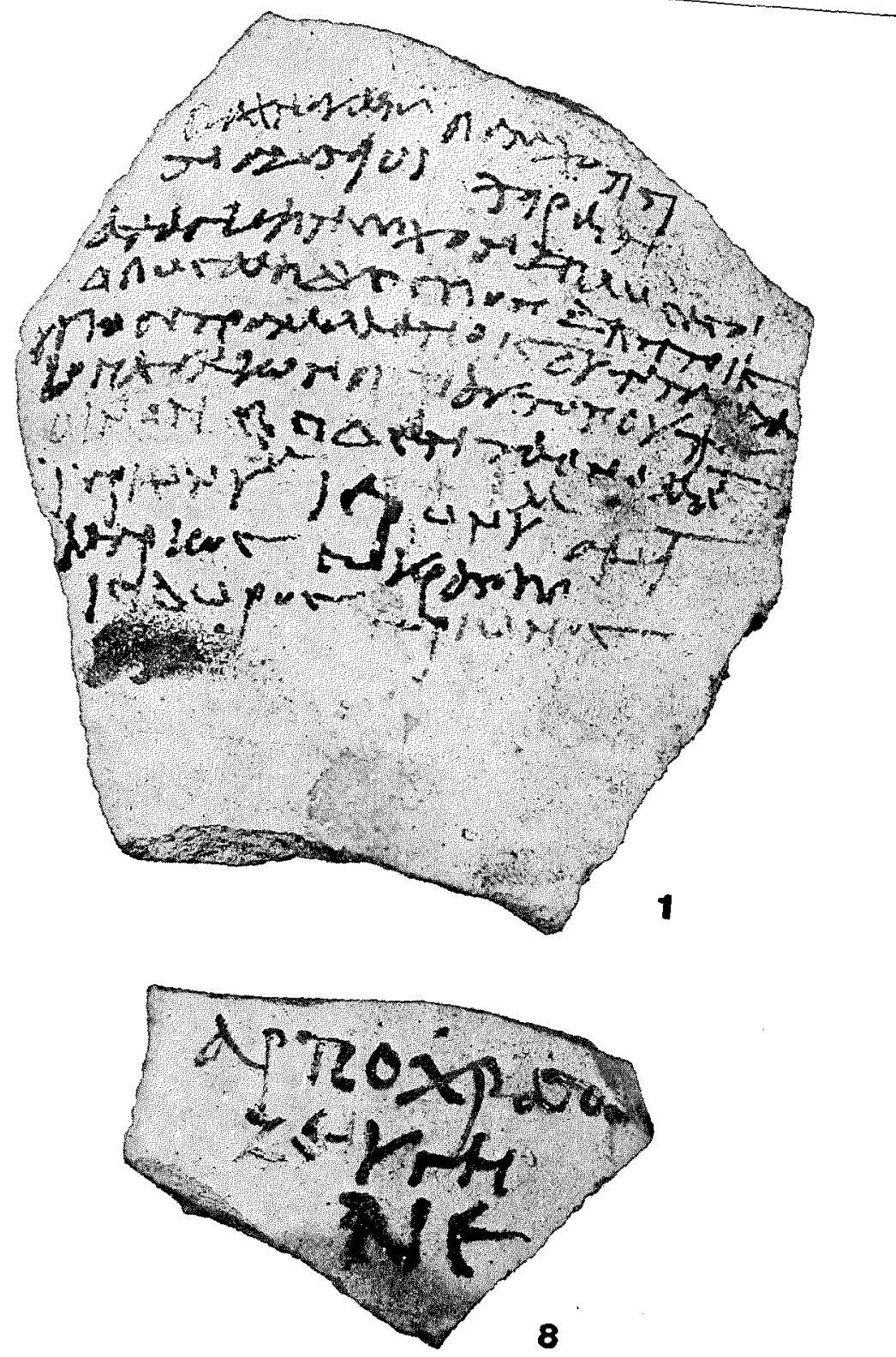

FxG. 1. - Ostraca 1 et 8. 
reçu ni argent ni vin à leur sujet. Voici leur nom : Hierônymos fils de Hierônymos, maître d'œuvre; Markos fils de Sôkratès; Isidôros fils d'Hôrion. 》

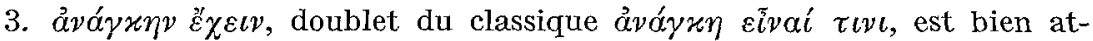
testé dans les lettres sur papyrus entre le $\mathrm{I}^{\mathrm{er}}$ et le vir $\mathrm{e}^{\mathrm{e}}$ s. p.C. ; $S B 7352$,

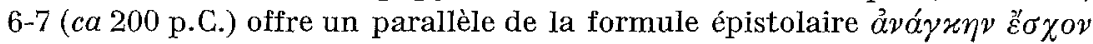
$\sigma o \iota \delta \eta \lambda \tilde{\omega} \sigma \alpha \iota$.

4. Surtaxe ou gratification, la $\sigma \pi \circ \nu \delta \eta$ est traditionnellement acquittée en vin ou en espèces (voir S. Ertrem, Symb. Osl. 17, 1937, pp. 27-41) : ici, les deux modes de paiement sont envisagés.

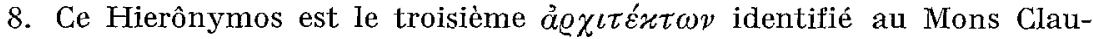
dianus; les deux autres, Hèrakleidès et l'Alexandrin Apollônios fils d'Ammônios, sont connus par des inscriptions (A. BERnand, Pan du désert, no 38 et 41 . Cf. Chron. Ég. 61, 1986, p. 146, pour le premier nommé). D'après Fitzler, les " architectes " des carrières dirigeaient le transport des blocs et peut-être aussi l'extraction (Steinbrüche, 133). Ce n'est sans doute pas un hasard si les agents payeurs et les bénéficiaires potentiels appartiennent à deux champs onomastiques distincts: Markos et Isidôros, comme leur collègue, ne devaient pas faire partie des couches les plus humbles parmi les travailleurs du Mons Claudianus.

\section{Deux fragments non ragcordables d'Une lettre.}

L'écriture et l'aspect du support ne laissent aucun doute sur l'appartenance des deux fragments à un même texte et la taille des lettres, que le scribe réduit progressivement, assure que le fragment $\alpha$ précède bien le fragment $b$. La moitié au moins du texte est perdue, mais les dernières lignes, complètes, font entrevoir les modalités du ravitaillement en eau. Pour l'écriture, cf. O. Amst. 69, pl. XIV.

a $5 \times 2,5 \mathrm{~cm}$.

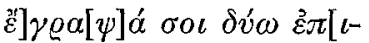

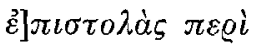

$$
\text { ]vo.. [ }
$$

b $7 \times 6 \mathrm{~cm}$.

$$
\begin{aligned}
& \text { env. } 9 \text { lettres } \quad] \alpha
\end{aligned}
$$

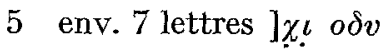

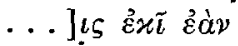

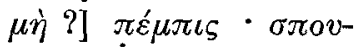

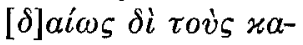




\section{$\mu \eta \lambda i ́ \tau \alpha \varsigma \mu \circ \iota \pi \dot{\varepsilon}-$ \\ 10

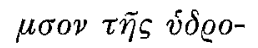 \\ pogías.}
1 l. $\delta \dot{v} o$
6 l. $\dot{\varepsilon} x \varepsilon \vec{\imath}$
7 1. $\pi \varepsilon ́ \mu \pi \eta S$
$8 l . \delta \eta^{\prime}$
9-10 l. $\pi \varepsilon ́ \mu \psi o v$

"... je t'ai écrit deux lettres au sujet de (?) ... là-bas si tu n'envoies pas (?) ... Dépêche-toi donc de m'envoyer les chameliers pour le transport de I'eau. »

5. La première lettre peut être aussi un $\lambda$; $\alpha$ me semble exclu. La deuxième, malgré les apparences, n'est pas un $\varrho$ : ce qui en paraît la boucle est une haste descendant de la ligne supérieure.

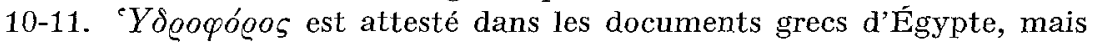

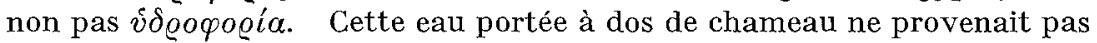
de la vallée du Nil, qui était à $140 \mathrm{~km}$. de là, mais des puits qui se trouvent à quelques $\mathrm{km}$. tout au plus des deux camps et dont le rendement devait suffire aux besoins des hommes et des bêtes, du travail et des cultures éventuelles $\left({ }^{1}\right)$. Notre texte résout, me semble-t-il, le problème logistique que pose la présence dans la station du wadi sud (alias l'" hydreuma ") d'une grande citerne dont Kraus estime la contenance à plus de 60.000 litres et à proximité de laquelle aucun point d'eau n'a été repéré : à l'ỉnstar des fontaines publiques de l'ancien Caire (sébils), elle devait être approvi-

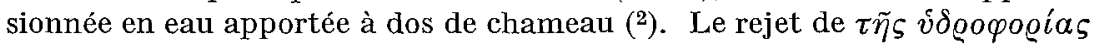

(1) J. Röder estime que dans l'antiquité, alors que les hydreumata, qui aujourd'hui encore ne sont pas à sec, fournissaient de l'eau en abondance, il a pu y avoir des champs, des vergers, des potagers sur le site ( $M D A I K 22$, p. 156). Des cultures à grande échelle sont évidemment exclues mais il est très probable que les hôtes $d u$ Claudianus ont produit sur place les fruits et les légumes frais qu'ils ne pouvaient faire venir, et ce peut-être dans des enclos analogues aux jardins bédouins de la région que l'on voit aujourd'hui verdoyer en plein désert autour d'un point d'eau (par exemple au débouché du Wadi Qattar). On imagine mal les privilégiés qui jouissaient du bain à l'ouest du camp, utilisaient la fine vaisselle pastillée dont on trouve des tessons sur le site, ou encore s'essayaient à composer des tirades en trimètres iambiques ( $Z P E$ 62, pp. 71-73), se passant de ces denrées. Au reste, la présence de cultures aux alentours des camps romains est un fait bien connu (ci. R. MacMullen, Soldier and Civilian in the Later Roman Empire, chap. I) et les wadi de ce massif montagneux offrent de la terre arable et parfois même se couvrent spontanément de végétation.

(2) Cette solution a été entrevue par Kraus qui, sans renoncer à l'hypothèse d'une alimentation de la citerne par les pluies, préfère considérer que l'eau venait du Wadi Umm Diqal voisin ( $M D A I K 22$, p. 137). Effectivement, Ie wadi où se trouve la station était relié au Wadi Umm Diqal par une piste qui aboutit précisément au niveau du grand puits antique; de cette piste subsiste aujourd'hui un long mur de pierre, haut de 0,50 m. env., dont on a longtemps cru qu'il s'agissait de l'aqueduc qui 
après l'impératif suggère peut-être que ce génitif est à comprendre comme

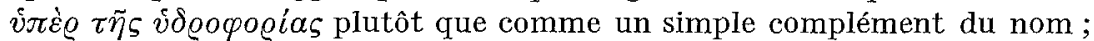
je ne vois cependant de cette omission de $i \pi \delta \dot{\varepsilon} \varrho$ que des exemples figurant dans des comptes, où le génitif désigne la raison d'un versement.

3. Fragment de Lettre. $5,5 \times 5,5 \mathrm{~cm}$.

Demande de ravitaillement. Pour l'écriture, cf. O. Amst. 70, pl. XV.

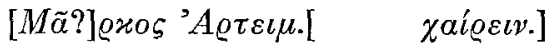

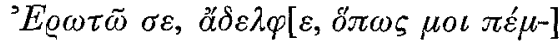

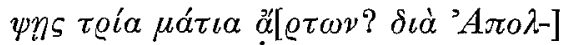

$$
\begin{aligned}
& \lambda \tilde{\omega} \tau o \varsigma \tau o \tilde{v} \varkappa \alpha \mu[\eta \lambda i \tau o v i v \alpha] \\
& 5 \quad[\mu] \dot{\eta} \pi \iota \mathcal{\omega} \mu \varepsilon v \quad .[ \\
& \text { ]. } \quad \ddot{\alpha} \delta \varepsilon \lambda[\varphi \varepsilon \\
& \text { 1 l. }{ }^{3} A \varrho \tau \varepsilon \mu[\quad 5 \text { l. } \pi \varepsilon เ \nu \tilde{\omega} \mu \varepsilon \nu
\end{aligned}
$$

"Markos à Artem [- salut.] Je te prie, frère, de m'envoyer trois matia de pains (?) par l'intermédiaire d'Apollôs le chamelier pour que nous ne mourions pas de faim... 》

2-3. La construction $\vec{\varepsilon} \varrho \omega \tau \tilde{\omega} \sigma \varepsilon+$ infinitif est beaucoup plus fréquente ; pour $\varepsilon \varrho \omega \tau \tilde{\omega}$ oह ó $\tau \omega \varsigma$ ou $i v \alpha+$ subjonctif $2^{\mathrm{e}}$ pers. sing. dans des lettres, cf. par exemple $P$. Tebt. 409, 4-8 et P. Fay. 113, 6-7.

3. Sur le $\mu a ́ t \iota o \nu$, voir ici même l'ostracon no 7,1 et le commentaire ad loc.

4. Fragment de lettre. $6 \times 3,5 \mathrm{~cm}$.

Manquent le haut, toute la partie droite et un morceau de la partie gauche. Il est question, semble-t-il, d'ânier(s) et de réserve(s) d'orge vide(s). Pour l'écriture, cf. O. Amst. 82.

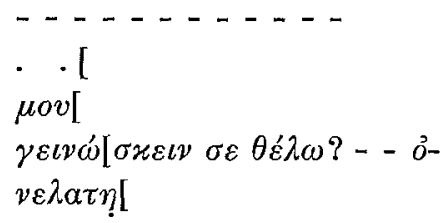

ravitaillait la station (réf. citées $M D A I K 18$, p. 98), supposition qui se heurte à plusieurs impossibilités : ce mur était vraisemblablement destiné à protéger la piste contre l'ensablement (MDAIK 22, p. 139). 


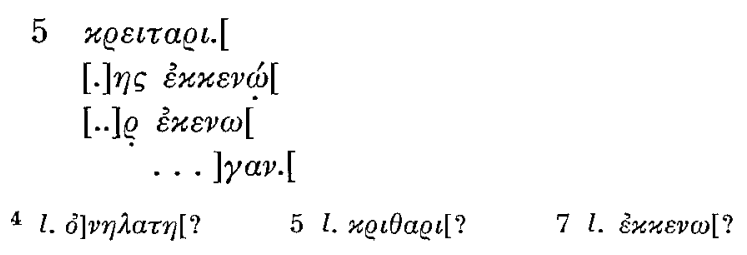

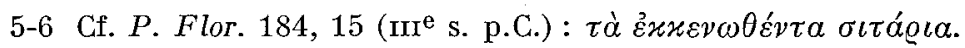

5. Fragment de letrre. $5,5 \times 5 \mathrm{~cm}$.

L'objet précis de cette lettre très mutilée (le support est brisé en haut, à gauche et peut-être aussi à droite, la surface est rongée par les sels) nous échappe. Cursive fine et expérimentée; $\eta$ en forme de y.

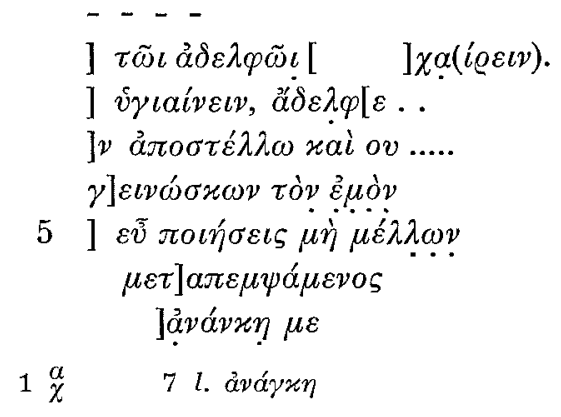

« $X$ à son frère $Y \ldots$ porte toi bien ... j'envoie et ... connaissant mon ... tu feras bien d'envoyer chercher sans tarder ... (car?) je suis contraint. " 5. $M \varepsilon \dot{\lambda} \lambda \varepsilon \varepsilon \imath$ au sens de "tarder» appartient plutôt à la langue poétique (cf. $L S J$ s.v.) et Preisigke, $W B$, n'en a pas relevé d'exemple.

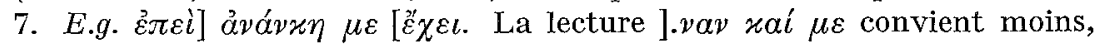
mais est possible.

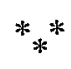

6-18. Dossier des couples de pains.

Ces douze ostraca sortent du même bureau : à deux variantes près, le schéma en est constant : un nom, parfois accompagné d'un patronyme,

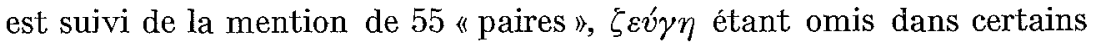
cas. La clef nous est fournie par l'inventaire étudié plus loin, où il est question de «couples de pains » (ó $\varrho \tau \omega \nu \zeta \varepsilon v ́ \gamma \eta) ;$ nos documents ne sont 
d'ailleurs pas les seuls à employer dans ce sens $\zeta \varepsilon v ́ \gamma \eta$ non précisé par $\not \varrho \varrho \tau \omega \nu$ : voir aussi $P$. Lond. 18, 7 ; WO 1209 et 1216.

Aux couples de pains, bien attestés dans les papyrus grecs d'Égypte à toutes les époques ( ${ }^{1}$ ), Wilcken consacre un développement sur lequel il n'y a pas à revenir (WO I 755-7 : l'artabe de grain étant convertible - à des taux variables - en couples de pains ( $P$. Lond. 18,$7 ; P$. Flor. 322,

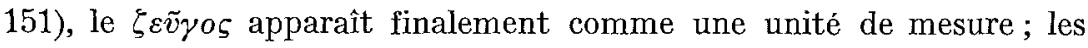
deux pains d'une même paire correspondaient à une certaine quantité de matière première.

Pourquoi cependant l'objet ainsi standardisé est-il non le pain, mais la paire de pains (au point que le rédacteur de $P$. Tebt. 253 inscrit dans sa comptabilité le prix de six paires et demie)? L'état de la documentation n'autorise aucune certitude, mais on peut supposer que ces deux pains (ou ce double pain) étaient, originellement au moins, destinés aux deux principaux repas d'une journée; le contexte indique en effet très souvent qu'ils sont distribués à titre de salaire ou d'allocation, ce qui est encore le cas jici.

Bien qu'on puisse, non sans difficulté, distinguer plusieurs mains, nos ostraca présentent une grande unité paléographique : l'écriture, sans être

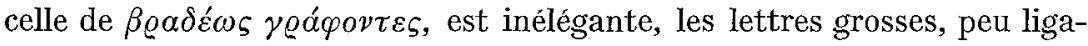
turées, le trait épais, l'encrage irrégulier. Plutôt que des ordres de paiement ou des reçus, dont la présentation serait moins informelle, j'y verrais volontiers des tickets alimentaires contre lesquels les porteurs se faisaient délivrer leur ration de plusieurs jours ou, peut-être, puisque les noms sont dans certains cas au génitif, des étiquettes qui, posées sur des sacs ou des paniers, servaient à identifier les rations revenant à des individus ou des unités.

6-11 (main I).

6. $5 \times 4,5 \mathrm{~cm}$.

$$
\begin{aligned}
& \text { 'Aло } \lambda \lambda \omega \nu(-) \\
& \Delta \text { covvoílov) } \\
& \nu \varepsilon
\end{aligned}
$$

7. $6 \times 5,5 \mathrm{~cm}$.

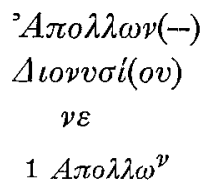

$1 A \pi \circ \lambda \lambda \omega^{\nu}$

(1) L'exemple le plus tardif est $B G U 2175,8\left(\mathrm{v}^{-} \mathrm{vI}^{\mathrm{e}} \mathrm{s}\right.$ ), où cinquante "paires de pains simples $»$ interviennent dans le paiement d'un loyer foncier. Je ne connais d'exemple de cette façon de compter le pain que dans la documentation grecque d'Égypte. 
«Apollôn(ios?) fils de Dionysios, 55. »

Il s'agit probablement du même bénéficiaire.

1. Il est difficile de décider si le point d'encre, peut-être parasite, sur le $v$ de 6 et la suspension du $\nu$ en 7 (attribuable aussi bien au manque de place), signalent ou non que le nom est un dérivé d' 'Aлó $\lambda \lambda \omega \nu$, mais le fait qu'on affronte le problème dans les deux textes plaide pour la dernière solution. 2. Un simple point, respectivement au-dessus du $\iota$ et en haut à droite marque l'abréviation dans les deux textes.

8. $7 \times 4 \mathrm{~cm}$. (fig. 1 ).

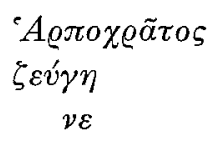

"D'Harpochras, 55 couples. "

1. Un nouvel avatar du nom Harpocratès? On songe évidemment au

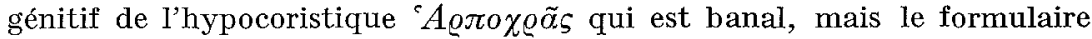
permet aussi le nominatif. Sur le génitif, voir plus haut, p. 278.

9. $6 \times 5,5 \mathrm{~cm}$.

$$
\begin{aligned}
& { }^{\circ} A \mu \mu \omega v i(o v) \\
& \zeta \varepsilon v \dot{\gamma} \eta \\
& \frac{\nu \varepsilon}{}
\end{aligned}
$$

«D'Ammônios, 55 couples. »
10. $2,5 \times 6,5$ cm. (fig. 2 ).

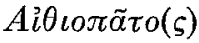
$\overline{\nu \varepsilon}$

"D'Aethiopas (?), 55 couples. »

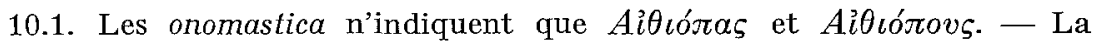
finale est susceptible de plusieurs lectures. La plus vraisemblable est celle que nous proposons, mais on peut songer à un $\alpha$ suivi d'un $\iota$ avec tréma empâté et d'un sigma ou d'un o surmonté des restes d'une barre (Aï $\theta_{\iota}$ $\pi$ á̈̈s?). Voir le commentaire du $\mathbf{n}^{0} \mathbf{8}$, sur l'emploi du génitif.

11 (main I?). $4,5 \times 3 \mathrm{~cm}$.

$\begin{array}{ll} & \Pi \varrho[ \\ & \sum_{\iota} \lambda .[ \\ & \zeta \varepsilon v \dot{\gamma}[(\eta) \nu \varepsilon]\end{array}$

$3 \zeta \varepsilon v^{\gamma}$

2. La barre verticale qui traverse la dernière lettre doit être parasite : à part une attestation douteuse dans Pape-Benseler, il n'existe pas d'anthroponymes commençant par $\Sigma_{\iota} \lambda \varphi$-. Plusieurs possibilités en revanche sont ouvertes avec $\Sigma_{\iota} \lambda o-$, au premier chef $\Sigma_{\iota} \lambda_{\text {ov }} \alpha$ vós, fréquent dans les carrières. 
12-15. (main II).

$$
\begin{aligned}
& \text { 12. } 7 \times 5 \mathrm{~cm} . \\
& \text { 'A } A \circ \lambda \lambda \omega \nu(\quad) ? \\
& { }^{\circ} O \nu \circ \tilde{v} \zeta \varepsilon v \dot{\gamma} \eta
\end{aligned}
$$

$v \varepsilon$

$1 A \tau \circ \lambda \lambda \omega^{\nu}$

"Apollôn(ios?) fils d'Onès, 55 couples. "

$$
\begin{gathered}
13 . \quad 5 \times 9 \mathrm{~cm} . \\
\Delta\llcorner o \sigma \varkappa(\text { ) } B o \theta v \dot{v}(o v) \\
\zeta \varepsilon v \dot{\gamma}(\eta) \nu \varepsilon \\
1 \Delta \iota \sigma^{x} \quad B o \theta v^{v} \quad 2 \zeta \varepsilon v \gamma \\
\text { «Diosk( ) fils de Bothynos, } 55 \text { couples.» }
\end{gathered}
$$

1. Le $\theta$, très empâté, pourrait passer pour un $o$. Ce patronyme est inconnu et doit être un sobriquet ( $\beta$ ó $\theta v$ vos signifie «trou $»)$.

14. $8,5 \times 6 \mathrm{~cm}$.

${ }^{\top} I \varepsilon \varrho \alpha \varkappa(\quad) \Phi \alpha \varkappa \tilde{\alpha} \zeta$

$\zeta \varepsilon \dot{\gamma} \gamma(\eta) \nu \varepsilon$

$1 I \varepsilon Q a^{\varkappa}$

l. $\Phi \alpha \propto \tilde{\alpha}$

15. $5 \times 4 \mathrm{~cm}$.

$--] \tau \alpha \varrho \chi \sigma \varsigma$

$\zeta \varepsilon] v \dot{\gamma}(\eta) \nu \varepsilon$

$21 v \gamma$

"Hierak( ) fils de Phakas, 55 couples."

14, 1. Le $\varkappa$ suspendu est si cursif qu'il affecte la forme d'un $\mathrm{L}$; celui de $\Delta \iota \sigma \sigma \varkappa($ ) dans le document précédent, presque aussi cursif, garantit la

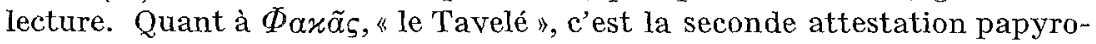
logique de ce sobriquet après $P$. Thmouis 146, 13.

16 (main III).

16. $5 \times 4 \mathrm{~cm}$.

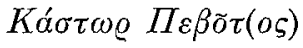

$\zeta \varepsilon v^{\prime} \gamma(\eta) \nu \varepsilon \sigma \varphi v \varrho i ́ \delta(t a)$

$\bar{\gamma} \mu \alpha \varrho \sigma \iota \pi[()]$.

1 l. $\Pi \varepsilon \beta \tilde{\omega} \tau(o \varsigma) \quad 2 \zeta \varepsilon v \gamma \quad \sigma \varphi v \varrho \delta$

"Kastôr fils de Pebôs, 55 couples, 3 paniers, $x$ sacs. " 
17-18 (main IV).

17. $6,5 \times 5 \mathrm{~cm}$.

C'est le seul exemple de la série établi au nom de deux attributaires ; le nombre de paires de pains est doublé, ce qui était attendu.

$$
\begin{gathered}
{\left[{ }^{[} E\right] \varrho \mu \sigma \gamma \varepsilon \dot{v} \eta \varsigma} \\
\varkappa \alpha i{ }^{~} H \varrho \omega \nu \zeta \varepsilon v(\gamma \eta) \\
\varrho \iota
\end{gathered}
$$

"Hermogénès et Hèron, 110 couples. »

18. $5 \times 4 \mathrm{~cm}$.

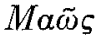

$\zeta \varepsilon v(\gamma \eta) \nu \delta$

$2 \delta$ écrit par-dessus le $\varepsilon$

«Maôs, 54 couples. »

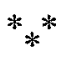

19. Inventaire. $8,5 \times 8 \mathrm{~cm}$. (fig. 2 ).

Si l'accusatif $\Delta \iota$ s'agit d'une liste de biens adressés à deux destinataires au moins. A comparer notre texte avec $P$. Oxy. 2424, on s'aperçoit que ces articles domestiques sont des plus courants : on retrouve dans cet inventaire sur

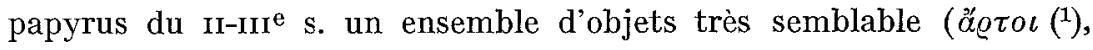

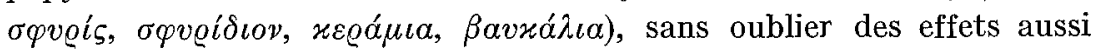
spécifiques que l'étrille et l'oreiller. Élégante semi-cursive (cf. O. Florida 19, pl. 10).

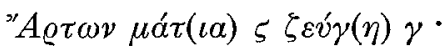

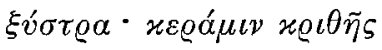

$$
\begin{aligned}
& \alpha \angle \cdot \ddot{\alpha} \lambda \lambda o \text { } x \varepsilon \varrho \alpha ́ \mu \iota \nu \text { } x \lambda \alpha \sigma \mu \alpha \alpha \tau(\omega \nu) \cdot \\
& \pi \varepsilon \dot{\tau} \tau \alpha \sigma o \nu \cdot \sigma \varphi v \varrho i \delta \iota v \cdot
\end{aligned}
$$

(1) Ne pourrait-on lire $\zeta \varepsilon v ́ \gamma \eta$ aux lignes 9-10 (cf. P.Lond. 190, 31-32, où sont également comptabilisées successivement des paires de pains et de gâteaux, $\lambda \alpha-$ $\gamma \alpha \dot{v} \iota \alpha) ?$ 
$5 x \varepsilon \varrho \beta \iota \alpha_{\alpha} \varrho \iota v \cdot x \alpha ́ \sigma \eta \pi \alpha-$

$\lambda \alpha \iota a ́ \cdot \theta \dot{\sigma} \sigma \alpha a \imath \delta \dot{w} \omega \cdot \beta a v-$

$\varkappa \alpha^{\prime} \lambda \varsigma s \delta v^{\prime} \omega$.

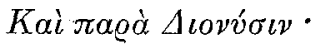

$\dot{\alpha} \sigma \tau i \delta \alpha \cdot \ddot{\alpha} \varrho \tau \omega \nu$

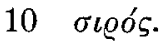

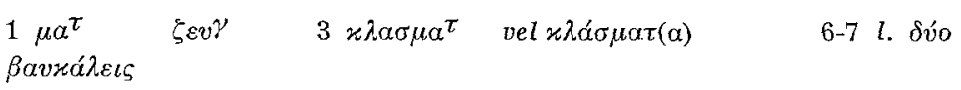

" 6 matia de pains, 3 couples; une étrille; $11 / 2$ keramion d'orge; un autre keramion de morceaux de pain ; un pétase ; un petit panier ; un oreiller ; un vieux casque ; deux brûle-parfums ; deux gargoulettes.

"Et chez Dionysios : un bouclier, une jarre de pains."

1. La mention de matia de pains, quoique nouvelle, n'est pas pour étonner: le mation est un sous-multiple de l'artabe, dont il représente le $1 / 12\left({ }^{1}\right)$, et j'ai pu relever quelques exemples d'artabes et de demi-artabes (=6 matia, comme ici) de pains ${ }^{(2)}$. La mesure s'applique-t-elle à la masse de pains ou bien au volume de grain entré dans la fabrication? Ce qu'on sait sur les équivalences établies parfois dans les documents entre artabes de grain et couples de pains (voir supra) invite à préférer la seconde hypothèse (qui nous donne en outre un ordre de grandeur pour la paire de pains, laquelle, d'après nos chiffres, est forcément inférieure à $1 / 36$ d'artabe); signalons cependant que le mation ne sert pas seulement à mesurer des céréales ou des épices : on connait des matia de dattes (e.g. P. Aberdeen 57, 19-20, $\mathrm{II}^{\mathrm{e}}$ s. p.C.) d'oignons (O. Fawakir $12,10=S B 9017$, $\mathrm{rI}^{\mathrm{e}}$ s. p.C.), de pommes (P.Giss.Univ. 25, 2, $\mathrm{III}^{\mathrm{e}} \mathrm{s}$. p.C.). 3 . Le sens premier de $x \lambda \alpha \dot{\alpha} \sigma \mu \alpha$ est "morceau, fragment». En papyrologie, on ne le rencontre que dans les comptes byzantins où il désigne une certaine forme de poste de paiement (voir Rémondon, $P$. Apoll. 100, 6 n.) $\left(^{3}\right)$; dans P.Lond. 1435,158 et 1443,65 , il est clair que $x \lambda \alpha \sigma \mu()$ annonce une liste d'entrées. Dans le même ordre d'idées, $x \lambda \alpha ́ \alpha \mu \alpha \tau(\alpha)$ n'aurait-il pas ici le sens, plus concret, d'" articles divers " (mais l'étrille aurait dû figurer parmi les miscellanea énumérés)? On est cependant

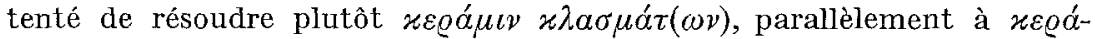
$\mu \iota \nu x_{\iota} \theta \tilde{\eta}_{5}$ : il s'agirait probablement alors de "morceaux de pain";

(1) Sur le mation, voir en dernier lieu S. P. Vheeming, Note on the maace, Enchoria 11 (1982), pp. 115-6.

(2) P.Ath. 14, 15 (22 p.C.) ; BGU 1095, 15 (57 p.C.) ; $B G U 538,33-34$ (100 p.C.) ; O.Stras. 474 et 475 (rve s. p.C.).

(3) $K \lambda \alpha \dot{\sigma} \sigma \mu \alpha$ n'a cependant pas ce sens dans son attestation papyrologique la plus ancienne, P.Mich. 741,6 (VI ${ }^{\mathrm{e}} \mathrm{s}$ ), où une Iivraison de vin est effectuée viż̀ $x \lambda \alpha ́ \sigma \mu \alpha(\tau \circ \varsigma)$. 

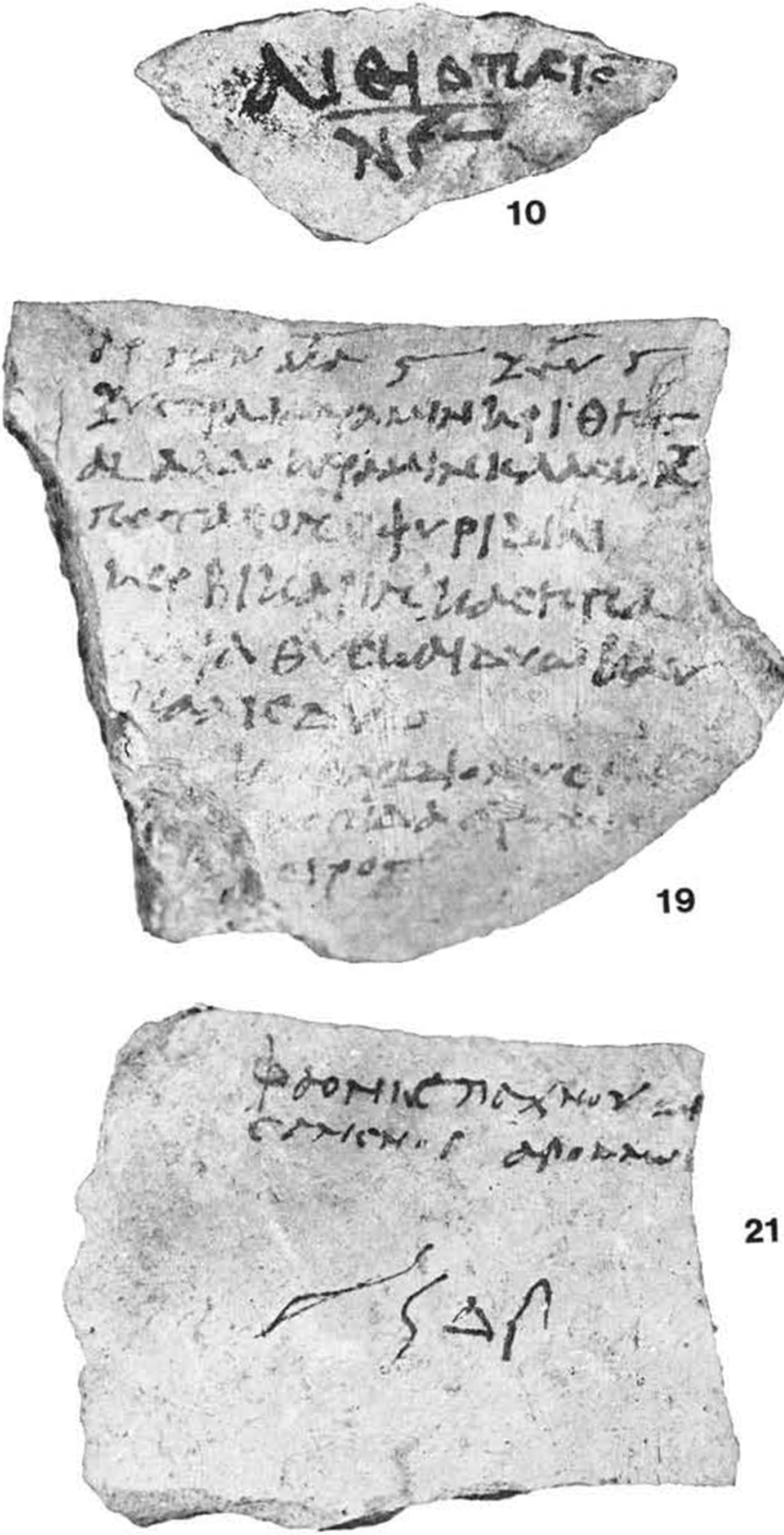

Fig. 2. - Ostraca 10, 19 et 21. 
ainsi sont désignés dans l'Évangile de Marc, les reliefs de la multiplication

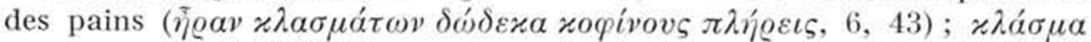
s'applique régulièrement au pain eucharistique (Lampe, s.v.) mais une épigramme de Lucillius, contemporain de Néron, emploie le terme dans

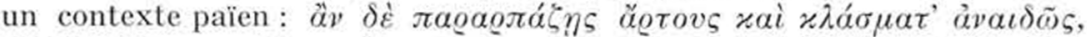
"mais si tu me chipes effrontément mes morceaux de pain ", Anth. Pal. 11, 153, trad. Aubreton. Dans l'Égypte contemporaine, on fait rassir ou sécher au four des pains ou des morceaux de pain, ce qui assure une conservation de longue durée.

4. Je ne connais pas d'autre attestation papyrologique du pétase que $U P Z 158 \mathrm{~A}, 108$. L'isolement, les longs parcours qu'il implique, le soleil accablant dans cet univers minéral où l'ombre est rare aux heures les plus chaudes, font de ce chapeau de campagne et de voyage un accessoire vestimentaire attendu au Mons Claudianus.

5. Noter l'emploi de termes latins, ceruical/ceruicarium et cassis; ce dernier n'est attesté que deux fois dans la documentation grecque d'Égyp-

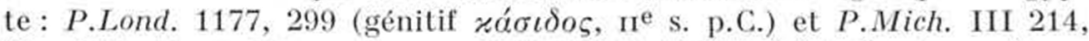
25 ; 216, 11 et 217, 17, trois lettres d'un même épistolier ( $\alpha \alpha \sigma \sigma i \delta \iota o \nu$, 296 p.C.). Le passage à la 1ère déclinaison greeque d'un substantif latin en -is n'est attesté en Égypte que pour classis : cf. les exemples réunis

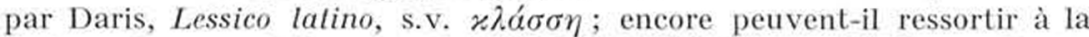
flexion du type $\theta \alpha ́ \lambda \alpha \tau \tau \alpha$, car le nominatif $x \lambda \alpha \sigma \sigma \eta$, déduit par Daris, n'apparait pas dans les textes tandis que l'accusatif $\varkappa \lambda \alpha \sigma \sigma \sigma \alpha \nu$ existe $\left.(S B 7354,6){ }^{(1}\right)$. Plus courante est la transposition en un diminutif neutre de la $2^{\mathrm{e}}$ décinaison, d'où $x \alpha \sigma \sigma i ́ \delta \iota \%$.

6. Un encensoir $\theta v ́ \sigma \varkappa \eta$ figure parmi des bagages de militaires dans $P$.

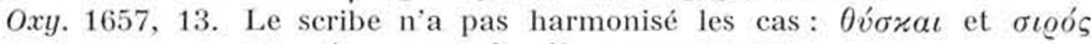
sont au nominatif, $\pi \varepsilon ́ \tau \alpha \sigma o \nu$ et $\ddot{\varepsilon} \sigma \pi \dot{\delta} \delta \alpha$ à l'accusatif.

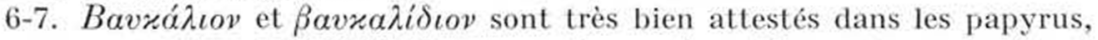
mais aucun des trois lexiques ne mentionne $\beta \alpha v \varkappa a ́ \lambda \iota \varsigma$ qui serait, d’après Athénée, le nom alexandrin d'un certain type de vase (XI 784 b).

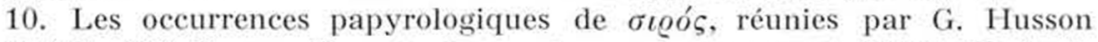
(Oikia, 252-3), ne démentent pas les explications de Columelle (voir ibid.), qui décrit une fosse creusée dans le sol et où l'on entreposait les céréales. En tout cas, les olgoí des papyrus ne semblent pas être des objets mobiles, comme c'est apparemment le cas ici où l'on imagine une grande jarre d'autant plus volontiers que cela concorde avec la définition $\pi i \theta$ os donnée par Hesychius. II est concevable au demeurant que le grain (et surtout l'eau; cf. G. Husson, o.c. 253, n. 2) n'était pas conservé à même la terre, mais dans de vastes pots enterrés; de là, le nom de

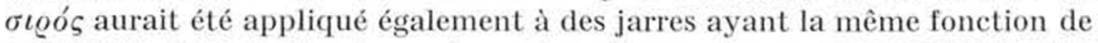

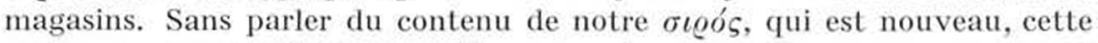
attestation est la première que l'on puisse localiser ailleurs qu'au Fayoum.

(1) Il en va de même pour vestis qui devient $\beta \dot{\varepsilon} \sigma \tau \eta$ : S. Daris se fonde sur un

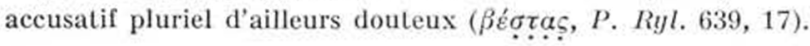


20. Liste DE QUATRE hommes. $8 \times 5 \mathrm{~cm}$.

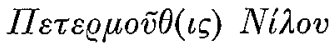

$$
\begin{aligned}
& \Pi \varepsilon \tau \varepsilon \varrho \mu о \tilde{v} \theta(\iota \varsigma) \Sigma \omega ́ \varrho o v
\end{aligned}
$$

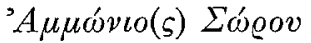

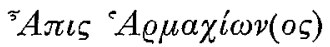

$$
\begin{aligned}
& 5 \\
& \bar{\delta} \\
& \text { 1-2 } \Pi 1 \varepsilon \varepsilon \varepsilon \mu o v \theta \quad 3 A \mu \mu \omega v \iota^{\circ} \quad 4 A \varrho \mu \alpha \chi \iota \omega^{\nu}
\end{aligned}
$$

"Petermouthis fils de Nilos; Petermouthis fils de Sôros; Ammônios fils de Sôros; Apis fils d'Harmachiôn : 4.»

1-2. Ou les doublets en $-\theta \eta \varsigma$ ou $-\theta \iota \iota$. La forme en $-\theta \iota \omega \nu$ est plus rare.

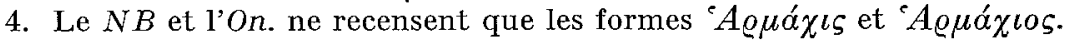

21. Attestation de paiement. $7 \times 6 \mathrm{~cm}$. (fig. 2).

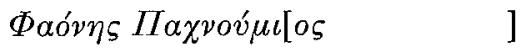

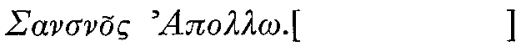

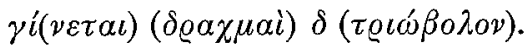

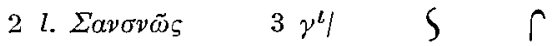

"Phaonès fils de Pachnoumis; Sansnôs fils d'Apollô[-]. Total : 4 drachmes 3 oboles. "

1. $\Phi \alpha \tilde{\omega} \nu$ est attesté, mais non $\Phi_{\alpha o ́} \nu \eta \zeta$.

22. Deux noms. $4,5 \times 3 \mathrm{~cm}$.

$$
\begin{aligned}
& \Phi \theta \circ \tilde{v} \varsigma \Pi \varepsilon \beta \tilde{\omega} \varsigma \\
& { }^{\prime} A \beta \tilde{\omega} \varsigma \Pi \varepsilon \beta \tilde{\omega} \varsigma
\end{aligned}
$$

"Phthous fils de Pebôs; Abôs fils de Pebôs."

1. $\Phi \theta \circ \tilde{v} \varsigma$ est nouveau à ma connaissance, mais $\Phi \theta \alpha \tilde{v} \varsigma$ et $\Phi \theta \varepsilon \tilde{v} \varsigma$ sont attestés.

23. Deux noms. $5,5 \times 4,5 \mathrm{~cm}$.

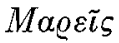

Teíovupos

"Mareis; Trioumphos. »

2. KaJanto, Latin Cognomina, 278, relève quatre attestations du cognomen Triumphus, jusqu'ici inconnu en Égypte. 
24. INSCRIPTION SUR JARRE. $8 \times 7 \mathrm{~cm}$.

$$
\begin{aligned}
& \text { 'A } A \pi \nu \lambda \lambda o[-- \\
& \text { 'A } \mu \mu \omega \nu[--
\end{aligned}
$$

25. INSCRIPTION SUR JARRE. $11 \times 10 \mathrm{~cm}$.

Ce récipient a dû contenir des olives. A la première ligne, un nom mutilé.

$$
\begin{aligned}
& \text { - - ]..ovil ) .[- - } \\
& \text { - - ] } \varepsilon^{2} \lambda \varepsilon^{\prime} \alpha \iota
\end{aligned}
$$

1. Le premier $\iota$ présente un empâtement qui peut le faire considérer comme un $\phi$. Un gros point sur le second $\iota$ indique sans doute que le mot est abrégé.

26. INSCRIPTION SUR JARRE. $12 \times 10 \mathrm{~cm}$.

\section{KQi $\sigma \pi \omega \iota$}

"Pour Krispos."

27. INSCRIPTION SUR JARRE. $9,5 \times 8,5 \mathrm{~cm}$.

$$
\text { Ỏंधน(- ) }
$$

On imagine difficilement ce qu'un vétéran serait venu faire au Mons Claudianus, et il doit s'agir simplement d'un anthroponyme latin en Vet-.

Le Caire, 5 mars 1986

Hélène Cuvigny 